\title{
Introduction : Conflits, territoires et action publique.
}

\author{
Patrice Melé
}

L'étude des conflits «territoriaux» peut-elle être une modalité privilégiée de l'analyse de la territorialisation de l'action publique ? Telle était l'interrogation à la base de cet ouvrage. Alors que les conflits peuvent être analysés du point de vue de l'action collective, des formes de mobilisation, des idéologies et des valeurs qu'ils portent, de l'organisation ou de la rationalité des habitants mobilisés, nous proposions de considérer leur dimension spatiale et territoriale. Ce texte ne vise pas à établir une synthèse des interventions ${ }^{2}$ du séminaire de l'équipe «Politiques publiques et territoires ». Il s'agit plutôt de proposer une lecture des différentes positions discutées dans ce séminaire et dans la littérature sur les conflits pour ensuite formuler des hypothèses sur le rôle des conflits dans les relations entre habitants et espace proche de leur logement, dans une certaine forme de production territoriale.

Il est possible de rendre compte de ces débats à partir de trois grandes interrogations : Que signifie parler de conflits territoriaux ? Que révèlent les situations de conflits? Quelles sont les dimensions territoriales des conflits et des controverses?

\footnotetext{
${ }^{1}$ géographe, VST, Université de Tours.

2 Les communications de Michel Lussault, «Micro conflits territoriaux et systèmes normatifs », Bernard Picon «Conflits d'usage de l'eau, imaginaire social et politiques publiques », Frédéric Poussin «La figuration comme support à la négociation du projet urbain » et Fabrice Ripoll «Quelles dimensions spatiales des actions collectives et des conflits sociaux?» absentes de cet ouvrage, sont néanmoins présentes dans cette conclusion à partir de références aux débats et de travaux antérieurs ou postérieurs à notre séminaire.
} 


\section{Conflits territoriaux, de quoi parle-t-on?}

\section{Différentes expressions d'oppositions, de revendications, de protestations}

Les figures du conflit mobilisées dans les recherches discutées lors du séminaire recouvrent différentes formes d'opposition :

- Des oppositions ou controverses entre habitants, usagers et acteurs publics : réactions de protestation face à un choix de localisation, à la réalisation d'une infrastructure [Lolive], à un projet ou un parti pris architectural [Rosemberg]. On peut classer dans ce type de conflit la question du contentieux mobilisé par des habitants [Lerousseau] ou certains micro-conflits liés à des pratiques de l'espace remises en cause par des projets publics [Lussautl]. Ces situations sont souvent nommées conflits environnementaux ou conflits d'implantation. Jean Marc Dziedzicki [2001 : 18-19] propose de distinguer des conflits d'aménagement s'opposant à la réalisation d'un projet, des conflits d'implantation liés à la perception des impacts potentiels d'un projet et des conflits d'environnement portant sur les impacts d'un équipement ou d'une activité en fonctionnement.

- Des oppositions, controverses, conflits entre usagers d'un espace. En étudiant la Camargue Bernard Picon [1988] a, par exemple, identifié des conflits d'échelles, d'ordres et de temporalités différentes. Celui ancien, juridicisé et mobilisant des groupes à fortes compétences et capacités d'investissement, lié à la gestion de l'eau et dont la résolution a produit l'équilibre précaire d'un milieu présenté comme nature; ceux plus récents qui mobilisent des références à une nature mythique et opposent différents groupes d'habitants à des projets ou pratiques allogènes. Dans un tout autre contexte, c'est le cas des conflits au Mexique entre habitants et lotisseurs sur les pentes des montagnes qui entourent la ville de Monterrey [Melé] et à proximité de Nantes celui de l'opposition d'habitants à l'implantation de porcheries industrielles dans l'espace péri-urbain [Rialland]. Les acteurs publics sont mis en cause pour avoir permis un certain type d'usage, n'avoir pas empêché un projet privé. Les valeurs environnementales sont mobilisées pour tenter de maintenir le statu quo et les qualités liées à certains usages ou à l'espace proche du logement. Ces situations peuvent être considérées comme des conflits d'usages.

- Des débats, oppositions entre acteurs publics ou para-publics lors de la négociation de projets ou la mise en œuvre de politiques publiques [Poussin]. Les relations entre acteurs dans le cadre de la production de l'action sur le territoire peuvent être de confrontation plus que de coopération, relever du rapport de force, de la résistance au changement ou simplement de négociations qui nécessitent la 
construction de situations publicisées comme opposition ou conflit. Il s'agit ici le plus souvent de différents ou de controverses - au sens d'interprétations contradictoires et d'argumentations fondées - entre des services ou des institutions qui peuvent être réglés par un accord négocié, un niveau supérieur administratif ou politique, ou se maintenir présentes révélant pour reprendre le vocabulaire de Michel Marié [1999 : 242], un «iceberg tensionnel» ou «des antagonismes réglés $»$.

- Des conflits sociaux et politiques analysés sous l'angle de leur dimension spatiale ou de leur relation au territoire. Luttes, mouvements [Ripoll] et oppositions électorales peuvent être lus à partir des espaces qu'ils mobilisent ou qualifient. La géographie électorale se propose de révéler l'inscription spatiale de rivalités politiques. Un effet de territoire dans lequel on pourrait lire la trace d'un conflit politique historique qui marquerait encore aujourd'hui les représentations et les positionnements politiques des populations [Roy].

\section{Situations qualifiées de conflictuelles}

De la simple existence d'antagonismes sur les visions ou les représentations du territoire et de l'action, à l'expression publique de désaccords, de controverses, des protestations d'habitants mobilisés dans le cadre de conflits d'usages à l'opposition à des projets publics, des manifestions jusqu'aux mouvements de masse, au contentieux et à l'expression électorale; les recherches présentées dans cet ouvrage qualifient de conflictuelles une grande diversité des situations.

Pour certains, le conflit apparait lorsqu'il est exprimé, médiatisé comme tel, pour d'autres, lorsqu'il est juridicisé [Lussault], une troisième position considère qu'il serait possible de parler de conflits pour toute expression d'antagonismes, de controverses ou d'opposition. Des intérêts divergents, compétitifs entre groupes sociaux, des représentations antagoniques peuvent être assimilés à des conflits que le chercheur pourrait révéler [D’Alessandro]. Plusieurs communications lors de notre séminaire considèrent les antagonismes de valeurs comme consubstantiels aux discours de l'action publique ou aux représentations de espace. Dans cette optique, ces antagonismes sont qualifiés de conflits, l'analyse du conflit étant dès lors déconnectée des moments de conflits. L'interprétation de ces antagonismes permettrait de révéler des tensions inhérentes au processus de territorialisation de l'action publique. Toute action sur le territoire, toute saisie d'un espace par un projet peut être considérée comme conflictuelle. L'analyse des antagonismes que révèle l'analyse des discours sert à mettre au jour les différentes valeurs présentes dans l'action sur le territoire [d'Alessandro ; Gravas, Veschambre]. 
Le séminaire a illustré la co-existence de différentes utilisations des notions d'antagonisme, de conflit ou de controverse. Or pour garder tout son intérêt à une approche par les conflits, il faut, me semble-t-il, réserver le mot conflit pour des situations qui constituent une manifestation de protestation ou d'opposition. Manifestation qui peut prendre la forme d'une stratégie de médiatisation, de dénonciation publique, d'actes de désobéissance civile, de troubles à l'ordre public et/ou de recours juridiques, autant de moyens de publicisation, constitution ou modification d'un rapport de force. Les conflits nous intéressent car, pendant ces manifestations, les acteurs développent des stratégies, proposent des discours, des visions du monde, des définitions de la situation qui permettent de construire un corpus susceptible d'être analysé. On aura compris qu'il ne s'agit pas de percer à jour les logiques des conflits par rapport aux évolutions structurales de la société ou aux modèles de la psychosociologie mais de prendre au sérieux le discours et les catégories des acteurs.

En cela, les phénomènes caractérisés par certains auteurs comme conflits latents, conflits sous-jacents, conflits non exprimés et qui renvoient soit à des analyses en terme de structures sociales, de structuration des représentations sociales, de psychologie sociale ou simplement d'usages potentiellement concurrentiels de certains espaces ne semblent pas relever du même type d'approche. Il ne s'agit pas de nier l'existence de tensions structurelles, d'antagonismes liés à des usages ou la validité d'explications psychologiques des relations interpersonnelles, mais simplement de souligner que ces tentatives se placent du côté de l'explication de l'existence des conflits, de l'identification de causalités. Alors que l'approche la plus féconde pour étudier les relations entre habitants, territoires et action publique s'avère celle de l'analyse des conflits comme situations, moments d'action collective dont il s'agit d'étudier les effets et la signification. Comme le note Lewis A. Coser [1982, 22]« il est essentiel de distinguer sentiments hostiles et conflit. Le conflit a toujours lieu dans une action réciproque entre deux ou plusieurs personnes. Les attitudes hostiles prédisposent à un comportement conflictuel; le conflit, au contraire est toujours une trans-action ».

Les notions de conflits latents, sous-jacents sont en particulier mobilisées pour tenter d'expliquer le caractère plus ou moins conflictuel de contextes locaux; pour répondre à la question: pourquoi un même projet d'aménagement ou certaines nuisances peuvent-ils être «acceptés » dans un contexte et l'objet d'un refus et d'un conflit dur dans un autre ?. Alors qu'il serait plus utile de caractériser des situations locales en terme d'antagonismes politiques ou de structuration sociale particulière plutôt que d'expliquer l'émergence d'un conflit par l'existence - d'un phénomène distinct puisque non manifeste - que l'on dénomme aussi conflit. La mobilisation des notions de conflits latents, sous-jacents ou de conflictualité 
potentielle du contexte local prend le risque d'expliquer des conflits manifestes par l'existence de conflits latents.

Les conflits sont donc particulièrement intéressants à étudier comme des moments de dramatisation du débat public. En effet, les acteurs mobilisés dans un conflit urbain doivent délimiter leurs positions en produisant un discours sur la justice ou la légitimité de l'action publique, sur l'intérêt général. Conflits et controverses instaurent une scène sur laquelle s'échangent des arguments [Trom, 1999: 34]. Ils rendent visibles les différents modes de justification à l'œuvre, les référents implicites ou explicites, et les représentations de l'espace urbain et de l'action publique. Le conflit « organise un temps pendant lequel les registres de légitimité sont rouverts $\gg$ [Fourniau, 1996].

Des controverses rendues publiques au conflit juridicisé, il existe une série de manifestations de dénonciation, opposition ou protestation, que l'on peut qualifier de conflictuelles. Ces moments de conflits qui constituent des phénomènes distincts peuvent être analysés comme des "situations". Analyser des situations ou des manifestations de conflit présente, à mon sens, l'avantage de ne pas réduire l'analyse à celle de tensions au sein ou entre groupes sociaux. De plus, partir de l'analyse de moments de conflits et de situations localisées permet de ne pas confier au chercheur le rôle de révéler des conflits latents sous-jacents ou potentiels.

Les conflits et controverses constituent des moments pendant lesquels différentes positions se présentent comme incompatibles. Or, ils se concluent le plus souvent par la construction d'une sortie négociée acceptable pour toutes les parties. L'exacerbation du conflit - qui porte la critique sur les opinions, les faits et les personnes - apparait alors comme intégrée dans une stratégie de constitution «d'un rapport de force, qui permet d'obtenir la victoire ou, à défaut, un équilibre qui justifie la négociation " [Meeus, 1997 : 46]. Dans la construction de ce rapport de force les signes médiatisés du conflit sont souvent aussi importants que l'ampleur de la mobilisation. Les signes de manifestation du conflit constituent dès lors des possibilités tactiques, répertoires et modes d'action à la disposition des promoteurs de l'action collective [Fillieule, 1993; Lafargue 1998].

Dans cette optique, il est possible de suivre Alberto Melucci [1999 :9] qui propose de considérer les situations de conflits comme des systèmes d'action et non comme des mouvements ayant une identité marquée par une essence, le type d'habitants mobilisés, la signification socio-politique qu'il est possible de leur attribuer dans un cadre d'analyse donné. Ce sont les processus d'implication, de mobilisation, de rapports entre les habitants mobilisés, mais aussi les relations externes avec des acteurs non mobilisés qui construisent ce système d'action. Si l'on considère les mobilisations 
comme des constructions sociales, résultats d'actions collectives, alors il devient important d'étudier comment les habitants définissent leurs actions. Les habitants mobilisés entrent dans le conflit avec leur vision du monde et parce que leur vision du monde les amène à considérer comme possible et pouvant porter ses fruits le type de mobilisation qu'ils mettent en œuvre. Les protestations collectives sont donc rendues possible par les attentes et les demandes ainsi que par les valeurs et croyances présentes dans la société. Les habitants mobilisés produisent l'action collective car s'ils sont capables de se définir et de définir le champ de leur action (relations avec les autres acteurs, disponibilité de ressources, opportunités et limitations) [Melucci, 1999 : 43].

\section{"Après le conflit, ce n'est pas la même chose qu'avant »}

Si le conflit est une situation, un moment, il est important de reprendre une idée présente plusieurs fois dans nos débats : «après le conflit, ce n'est pas la même chose qu'avant ». Il faut dès lors s'interroger sur les effets du moment de conflit pour les acteurs mobilisés. On peut émettre l'hypothèse que le moment du conflit modifie la perception/représentation d'un projet, des autorités publiques en cause, d'un espace présenté comme à préserver. Modification qui ne concerne pas seulement la position des acteurs insérés dans le conflit, mais aussi la façon dont des acteurs institutionnels extérieurs ou des habitants non mobilisés perçoivent l'espace qui peut apparaître dès lors comme enjeu, support d'appropriation et de projets distincts.

Analyser des conflits mobilisant des habitants contre des projets permet aussi d'introduire une interrogation sur l'évolution des relations entre habitants et action publique. On peut en effet considérer le conflit comme un moment d'apprentissage, d'exposition des habitants à la règle de droit et au système politico-administratif comme des moments de socialisation politique et juridique. Cette thématique est présente dans les recherches sur la professionnalisation des leaders et des militants, sur leurs rapports aux acteurs politiques, sur les transformations des représentations et des visions du monde d'habitants mobilisés et/ou insérés dans des processus de participation.

En ce sens, le moment du conflit n'est pas seulement un moment d'énonciation permettant de révéler des éléments du contexte social et politique, de la socialisation politique et juridique ou de l'ordre dogmatique préexistant, mais un moment de sa production. Les mobilisations d'habitants sur des thématiques de proximité sont souvent l'occasion pour les habitants de prendre conscience $\mathrm{du}$ fonctionnement de l'appareil politicoadministratif, d'approcher les réseaux d'intérêts croisés sur le territoire [Micoud, 2000 : 61]. Les situations de conflits sont aussi dotées d'un rôle de structuration sociale. Elles produisent associations, alliances et coalitions multipliant interactions entre participants. Le conflit peut donc être 
considéré comme un mécanisme qui «réduit l'isolement social» qui « rassemble des parties » [Coser, 1982].

\section{Conflits et controverses comme révélateurs}

Pour les chercheurs les situations de conflits sont souvent considérées comme des révélateurs. Le conflit devient un « analyseur social » [Charlier, 1999 : 88]. Les mobilisations se présentent aussi comme des dénonciations, des protestations rendues publiques. Les promoteurs de l'action collective proposent comme stratégie d'élargissement de leur base et de modification des rapports de force la révélation à l'opinion publique de leur vision des impacts d'une décision, projets ou action publique, le dévoilement des stratégies ou d'intérêts cachés.

Dans une perspective de changement social, les conflits qualifiés de (nouveaux) mouvements sociaux sont dotés de la capacité de rendre public ce que le système voudrait cacher. Non seulement les conflits diffusent des messages au reste de la société, relient un problème particulier à « la logique du système » mais par leur existence même proclament la possibilité d'une action, l'existence d'un modèle alternatif d'action ou d'implication de la population [Melucci 1999 : 126-127].

Une partie importante des travaux de sociologie de l'action collective s'intéressent à la définition structurelle ou à la recherche des causes structurelles de l'action collective [Melucci, 1999]. Les conflits ont souvent été analysés comme mouvements sociaux du point de vue des conditions sociales ou politiques des groupes qui les portent. L'analyse de ces conditions permettrait de déterminer les «causes » des conflits, qui peuvent être cherché dans la place de certaines couches sociales ou groupes exclus ou marginalisés de la représentation politique. Dans une perspective d'analyse des rapports de forces ou de distribution de ressources au sein de la société, de demande de citoyenneté mais aussi pratiquement dans le cadre d'un modèle de psychologie sociale où la frustration d'aspirations collectives déboucherait sur l'expression de conflits ou de mouvement sociaux; les situations de conflits sont interprétées comme révélant une situation politique, les aspirations ou attentes ou la résistance au changement de certains groupes sociaux.

Certains auteurs soulignent le rôle du droit dans la formalisation des attentes comme dans les représentations de la justice de la répartition entre nuisances et avantages liés à des positions spatiales. Dans cette optique, les conflits sont rendus possible à la fois par des attentes construites sur une vision du monde rendue perceptible par le droit, et par le droit qui crée ou vient conforter des attentes. En ce sens, l'accroissement des contentieux serait non pas une cause mais bien une conséquence de la juridicisation de la société [Gessner, 1993]. De nombreux conflits possèdent une dimension 
d'expression d'un sentiment d'injustice, d'une demande de validité des règles de droit, de revendication de «droits à ». Si la fonction dogmatique qu'évoque Pierre Legendre [1999] est constitutive de l'individu alors la question de «faire valoir son droit» n'est jamais une question technique impliquant seulement une rationalité limitée et une capacité à mobiliser une connaissance d'un droit particulier. Comme le soulignait déjà Georg Simmel [1995 : 48 ] la mobilisation du droit, les références à la justice impliquent la personne. Conflits et mobilisations sociales proclament souvent l'émergence d'un «sens différents de la justice» [Fillieule, Péchu, 1993 : 66]. Catherine Zwetkoff a mis en évidence le caractère ambivalent de l'ancrage dans des valeurs, de la mobilisation de principes de justice divergents, qui par leur capacité même de mobilisation rendent plus complexe une sortie négociée du conflit [Zwetkoff, $1997: 8$ ].

Les conflits peuvent aussi être saisis à partir de leur rôle de sensibilisation, de «concernement» du public, de formation de l'opinion publique, élément à distinguer de l'implication ou de la mobilisation dans le cadre d'une stratégie d'élargissement de la base des collectifs : "Depuis l'étude du concernement dans les associations, il faut passer à celle du concernement par les associations. C'est ce qui se dit notamment dans cette expression aussi utilisée (par toutes les associations sans exception), qu'elle est peu analysée: la nécessité de sensibiliser le public » [Micoud, Charvolin : 2000: 73]. Les associations de protection de la nature ou du patrimoine placent au premier plan ce travail de concernement.

Lorsqu'ils sont constitués sur la base de la lutte contre des mutations ou des nuisances du cadre de vie, les conflits se caractérisent par une "montée en généralité » mobilisant des conceptions de l'espace urbain, de la vie en ville, de l'action publique en ville, de la place du droit dans l'aménagement urbain. Soumis à l'impératif de démontrer que leur mouvement dépasse leur intérêt propre et ne peut simplement être classé - déqualifié - dans la catégorie NIMBY [Trom, 1999], les habitants mobilisés produisent un discours de justification ancrant leur mouvement dans des grandeurs, convoquant des valeurs, dont ils se proclament porte parole.

Les situations de conflits peuvent être considérées comme des moments de controverses se traduisant par une production d'actes, de discours, de figurations susceptibles d'analyses permettant de révéler les identités, qualités et compétences des acteurs. Dans cette optique les conflits et controverses seraient des révélateurs :

- des rapports de force, puisqu'ils obligent les pouvoirs et les porteurs d'intérêts à se montrer, à prendre une position [Melucci, 1999: 9],

- des relations des populations à l'espace, de phénomènes de territorialité ou d'appropriation, des représentations de la ville, des positions sur un projet urbain et sur l'avenir de la ville [Rosemberg], 
- de volontés d'appropriation réelle ou symbolique de l'espace par certains groupes sociaux [Gravas, Veschambre],

- des compétences et des ressources des habitants mobilisés, de leurs stratégies et répertoires d'action, de leur capacité à investir les réseaux d'action publique, à se construire comme experts des règles d'urbanisme, à mobiliser le droit [Lerousseau],

- de processus de montée en généralité qui mobilisent des valeurs permettant de mettre en débat l'utilité publique des projets [Melé].

D’une façon assez classique les conflits ont été analysés comme mettant au jour des dysfonctionnements de l'action publique :

- dysfonctionnements au sens de retards, de difficultés à mettre en œuvre un projet doté d'un degré d'acceptabilité minimale ;

- dysfonctionnement au sens d'une incapacité de l'action publique et des processus de concertation et participation à intégrer les demandes, attentes, revendications des populations. Non prises en comptes ces demandes, cette volonté de participation, s'exprimeraient dans des conflits ;

- dysfonctionnement au sens aussi d'une insuffisante prise en compte des impacts environnementaux et de la perception de pollution ou de risques potentiels ;

- dysfonctionnement produit par une «politisation» du débat sur l'action publique. Les conflits d'aménagement cacheraient alors l'expression d'opposants politiciens.

Pour Jean-Marc Dziedzicki [2001, 163-164] qui cherche à contribuer à la production de modalités de gestion des situations de conflits, l'attention doit donc se porter sur le rôle de «mécanismes conflictuels » à chercher du côte de la complexité de la situation et des prises de décision, d'une dynamique $\mathrm{du}$ conflit entretenue par des aspects relationnels, ou de controverses antérieures liées aux usages et aux représentations de l'espace support de l'aménagement. Dans cette optique l'analyse porte sur les causes des conflits, que l'on explique par les dysfonctionnements des décisions d'aménagement ou par des phénomènes psychologiques, ou de l'appropriation de l'espace. Des processus de négociation en amont, de participation, de concertation, de médiation visant la réduction de ces mécanismes conflictuels pourraient permettre de limiter l'impact des conflits.

Au-delà de cette identification de dysfonctionnements, les conflits peuvent être dotés aussi d'une fonction d'innovation, de repositionnement des acteurs, de construction d'un nouveau consensus territorial. Certaines recherches se focalisent sur les conditions dans lesquelles les conflits produisent de l'innovation. Dans cette optique le problème des aménageurs 
ne serait pas de limiter les conflits mais de contrôler les dynamiques destructives du conflit [Zwetkoff, 1997: 5]. Mettant à jour les dysfonctionnements de l'action publique, les conflits et controverses auraient une fonction d'alerte [Coser, 1982], un "pouvoir fertilisant » [Callon, Lascoumes, Barthe, 2001 : 24], permettraient la modernisation de l'action publique, l'intégration de nouvelles pratiques au sein des institutions, la mise en œuvre d'alliances et de nouvelles relations négociées entre acteurs.

Les conflits peuvent être dotés de la capacité d'améliorer des projets de faire émerger de nouvelles solutions, de susciter une meilleure prise en compte de l'environnement et des relations avec les populations. Certaines recherches se concentrent sur la prise de décision dans le cadre du conflit, sur l'adaptation des formes de l'action publique aux situations de conflits sur la mise en œuvre de processus de concertation et de gestion du conflit, évoluant vers une gestion négociée de l'espace, une construction locale de l'accord.

L'attention peut se porter sur la construction de l'assentiment, sur la construction de l'acceptable dans une optique de réduction des conflits ou de production de médiation [Dziedzicki, 2001], mais aussi comme le propose Jacques Ion [2000: 2] sur les « processus par lesquels une expression, une revendication, une mobilisation, portées par un ou des groupes spécifiques, parviennent à se constituer, à se formaliser, à s'énoncer et à accéder à l'espace public ».

\section{Dimensions spatiales et territoriales des conflits et controverses}

Des mobilisations sociales sectorielles peuvent être étudiées à partir de leurs dimensions spatiales [Ripoll] : stratégies de prise de la rue, choix de localisation d'actions, ancrages et bases locales. Les militants savent bien que certains contextes locaux peuvent être propices à des mobilisations. Les analyses de conflits sociaux relèvent l'utilisation stratégique de l'espace dans des «technologies d'action collective»; "technologie de l'encombrement urbain des chauffeurs » ou des habitants mobilisés, "stratégie de prise symbolique de l'espace urbain », blocage du centre-ville, barrages [Courty, 1993]. La manifestation de rue présente une spatialité et une temporalité interne [Fillieule, 1993].

Mais l'espace peut être plus directement l'objet du conflit. Dans le cadre de conflits d'usage ou d'appropriation opposant diverses formes de pratiques, l'espace constitue souvent explicitement ou implicitement cet objet commun, enjeu des disputes, nécessaire au déclenchement des conflits. Une situation de conflit permet aussi de mettre au jour les différents intérêts qui s'opposent sur un espace. Analyser les discours d'acteurs qui se proclament porteurs d'intérêts légitimes permet d'appréhender les stratégies, 
arrangements, discours et alliances à la base de la constitution de contextes locaux d'action.

Dans certains conflits qui revendiquent la protection de l'environnement ou du cadre vie, l'enjeu est le maintien de caractéristiques d'espaces urbains, péri-urbains ou ruraux, considérées comme constitutives des valeurs liées à cet espace. Certains types de conflits environnementaux peuvent être caractérisés comme portant sur l'espace en lui-même « en tant qu'il est doté de qualités propres » [Trom, 1999: 33]. Dans le cadre de conflits d'implantation ou de conflits liés à un projet d'aménagement, les controverses portent sur des visions antagonistes des usages légitimes, du l'organisation spatiale future, sur l'impact des actions publiques en débat. Sous le nom de conflit de territoire, Jean Marc Dziedzicki [2001:163] propose de prendre en compte deux thèmes conflictuels des conflits liés à un projet d'aménagement : celui des représentations individuelles et collectives de l'espace d'implantation, et celui des inquiétudes portant sur les impacts réels et potentiels de l'aménagement sur l'environnement.

Les discours et procédures de justification de l'entrée en conflit et de la légitimité des revendications mobilisent des visions du territoire, mais aussi des attentes, des argumentations sur les modalités de l'insertion et la position spatiale occupée par certains habitants. C'est ce que montre l'étude des conflits à Monterrey [Melé], mais aussi les conflits déclenchés par la possibilité d'une modification de la répartition spatiale des nuisances aéroportuaires autour de Lyon-Saint-Exupéry. Ce conflit exprime, avant toute nouvelle répartition spatiale des nuisances, la crainte de la remise en cause du calcul d'acceptabilité de leur position face aux nuisances de l'aéroport effectué par des propriétaires de pavillons venus s'implanter après la construction de l'aéroport [Melé, 2001]. Certaines revendications reposent sur la perception ou la construction de valeurs, de vulnérabilité ou de risques liés à des espaces particuliers.

Pour les habitants mobilisés dans un conflit pour la protection de l'espace proche de leur logement, les situations de conflits peuvent être vécues comme des moments de territorialisation: au sens de la définition d'une appartenance territoriale, d'identification et d'utilisation stratégique des valeurs qui peuvent être liées à certains espaces. Pour pouvoir être préservé et devenir enjeu de protection et de négociation, le « cadre de vie » doit faire l'objet d'une définition territoriale ${ }^{3}$, ses qualités d'une qualification

\footnotetext{
3 Je retiens ici la définition du territoire proposée par Jacques Lévy [1994: 76], assez proche de la définition juridique et politique d'espace délimité [Di Méo, 1996], qui présente l'intérêt de décrire un type de configuration spatiale sans recourir à la notion d'appropriation. Sous cette acception, le territoire - qui s'oppose au réseau - est un type particulier d'espace caractérisé par l'exhaustivité (à l'intérieur du territoire tout point se trouve dans le territoire) et la contiguïté (tout point du territoire est entouré de points du territoire sauf s'il se trouve sur les frontières du territoire)
} 
juridique. Cependant, comme le souligne Bruno Charlier [1999, 511], l'espace de servitude n'est pas isotrope, mais perçu à partir d'anamorphoses spatiales dont le rôle est important dans la conflictualité environnementale.

Les habitants se mobilisent pour l'affirmation d'un droit de regard sur les usages et pratiques de l'espace, proclamant un intérêt légitime à agir - de façon indépendante de sa reconnaissance en droit positif - . Ces phénomènes doivent-ils être considérés comme la construction d'une identité territoriale?. Ils permettent en tout cas la mise en évidence du rôle de l'espace dans la constitution d'une identité collective ou de "minorités » pour reprendre le vocabulaire de Michel Callon, Pierre Lascoumes et Yannick Barthe: «Des minorités (qui)..dans les exemples proposés, s'appellent myopathes, malades atteints du SIDA, riverains des sites de stockages de déchets nucléaires, populations habitant dans la proximité d'une usine chimique ou d'une décharge de produits toxiques, consommateurs de nourriture transgénique. Des minorités qui comme toutes les minorités, qu'elles soient ethniques ou religieuses, se battent pour se faire reconnaitre et entendre, se mobilisent pour être représentées » [Callon, Lascoumes, Barthe, 2001 : 329].

Une des fonctions sociales du conflit est de permettre de "souder des groupes » de susciter ou de modifier des communautés d'intérêt, de constituer une forme positive de socialisation [Simmel, 1995 ; Coser, 1982]. Dans le cas des conflits pour la protection du cadre de vie, de sousensembles spatiaux dotés de qualités environnementales ou contre un projet d'aménagement, ces groupes ont une base territoriale. Dans certains contextes, le conflit peut unir des habitants et usagers d'un espace à partir d'une coalition d'intérêt à fort ancrage spatial entre habitants et usagers. Ces conflits peuvent donc être considérés comme permettant la constitution d'un collectif produisant une vision territoriale de son ancrage spatial.

Si l'espace peut être enjeu, cadre de la définition d'une appartenance, il peut être aussi une ressource mobilisée pour justifier certaines positions. Des projets sont remis en cause au nom d'une valeur particulière : patrimoniale, environnementale ou de biodiversité. Ces valeurs peuvent être mobilisées pour protester contre des dynamiques susceptibles de modifier les relations privilégiées de certains habitants avec des espaces naturels à préserver ou simplement un paysage particulier [Melé].

Selon le principe d'identité construit par Alain Touraine [1973, 363] pour la caractérisation des mouvements sociaux, «c'est le conflit qui constitue et organise l'acteur ", au sens ou le conflit constitue un acteur collectif conscient des enjeux du conflit. Il est, me semble-t-il, possible de se référer à cette capacité des conflits même pour des mobilisations qui dans la terminologie d'Alain Touraine seraient dénommées conduites collectives, luttes ou même anti-mouvements sociaux [Touraine, 1984]. S'il n'y a pas ici 
lutte pour le contrôle de l'historicité, pour le contrôle de la société, on peut poser comme hypothèse la constitution d'un acteur collectif. Cette position est d'ailleurs assez proche de ce qu'Alberto Melucci [1999:66] analyse comme identité collective c'est-à-dire la capacité collective - construite dans l'interaction - de se définir soi-même et le contexte, d'évaluer les opportunités et les limites de l'action.

Lors de conflits d'implantation, d'usages ou d'environnement, la constitution de l'acteur collectif comme la définition des buts du conflit ont une base spatiale. Il serait d'ailleurs peut-être possible de considérer que la mobilisation a une base spatiale mais que le processus de délimitation des buts du conflit implique un travail collectif sur les représentations des usages légitimes de l'espace et une définition territoriale d'un espace à protéger, à préserver de certaines dynamiques.

Dans d'autres cas, l'espace du projet peut devenir l'espace de contestation, de mobilisation, des habitants se trouvent réunis dans une position nouvelle celle de « riverains » d'un projet, d'une infrastructure qui n'existe pas mais qui est perçue comme menaçante. C'est pourquoi la multiplication des variantes - aux tracés, à la localisation - lors des négociations d'un projet a souvent pour conséquence de multiplier les opposants potentiels [Lolive, 1999 ; Charlier, 1999].

Certains habitants se pensent inclus dans un territoire potentiel de nuisance ou de pollution. Il est donc possible de considérer les mobilisations contre un aménagement ou pour la sauvegarde d'un espace comme l'émergence d'une vision territoriale de l'ancrage spatial. Le périmètre du projet en cause peut devenir le périmètre de mobilisation. L'intéressement des populations à partir de leur logement et leur participation à une action collective passe par la reconnaissance de leur solidarité de destin avec d'autres habitants proches. La définition de ce groupe d'acteurs affectés constituant la base de la mobilisation potentielle est le plus souvent territoriale.

Jacques Lolive évoque un autre processus impliquant une relation au territoire sous le nom de «territorialisation réactive»: la valorisation d'éléments territoriaux menacés et la mobilisation de topiques dans la construction d'une argumentation contre un projet. Les valeurs attachées à des lieux deviennent des éléments d'argumentation contre un projet [Lolive, 1999]. Pour les habitants mobilisés ce moment de l'argumentation stratégique est aussi le moment de l'identification, de la reconnaissance ou de la production de «qualités » particulières attachées à des lieux ou des configurations spatiales. Cette valorisation de l'espace peut passer dans le cadre de processus de patrimonialisation par la promotion de la qualité particulière du milieu, du paysage, de certaines pratiques ou modes d'occupation de l'espace. 
L'implication dans des controverses et conflits peut être considérée comme un mode particulier de relation entre habitants et l'espace proche de leur logement. Pour tenter de s'imposer comme porte-parole légitimes des valeurs de l'espace à préserver, des habitants ont à construire une vision communicable de leur expérience sensible, celle-ci prenant le plus souvent une dimension territoriale et pouvant déboucher sur une demande de protection par la mise en place de qualifications juridiques spécifiques ou de structures de gestion offrant les assurances d'une vigilance renforcée.

Non seulement le conflit contribue à former des groupes sur une base spatiale ou territoriale, mais on peut dire que les arguments et les pratiques mises en œuvre par les habitants mobilisés contribuent à souder les groupes autour d'une certaine vision de l'espace, d'une certaine conception des valeurs de l'espace proche, construites au moment où il faut le défendre.

Cette production territoriale issue de situations de conflit doit-elle être considérée limitée dans le temps et à certaines pratiques [Lolive], ou les moments de conflits ont-ils la capacité de marquer durablement des espaces, de modifier les valeurs et représentations. Comme pour les politiques urbaines [Lussautl, 2001], la dimension temporelle des conflits relève du récit et de la mise en intrigue. Pendant le temps de l'action ou celui du récit les conflits qualifient des espaces. Ce qui a changé après le conflit, c'est non seulement la mise en réseau d'habitants, la capacité de certains d'entre eux à affronter les épreuves de la mobilisation et éventuellement leur promotion comme porte-parole du « territoire ». Ce qui a changé après le conflit, c'est que le territoire a été mobilisé. Il faudrait alors s'intéresser sur ce qu'il reste de cette mobilisation pour les habitants impliqués et pour les autres. Doit-on évoquer une mémoire des «territoires », sur le modèle du trauma territorial historique discuté par Jean Philippe Roy ou simplement considérer l'existence de processus de territorialisation résultant des mémoires, des récits des conflits et mobilisations? Les réseaux qui ont permis la mobilisation passée, ou simplement l'évocation de la capacité d'action sur le territoire d'une mobilisation « réussie » peuvent-ils être réactivés, constituer un contexte dans lequel perdure une vigilance particulière? 


\section{Conclusion : Conflits et controverses comme mode de territorialisation de l'action publique}

A l'image des mouvements sociaux non liés à un territoire - mouvement syndical, mouvement politique, mouvement de minorités culturelles - les conflits «territoriaux » portent une charge critique sur le fonctionnement de la démocratie représentative. Ils exprimeraient la non prise en compte adéquate, de positions, d'intérêts, de revendications. Dans le domaine de l'aménagement ou des conflits d'implantation, c'est le fonctionnement de la prise de décision technico-politique qui apparaît remis en cause.

La généralisation des controverses et conflits liés à des projets publics donne lieu à des interprétations opposées. Pour certains, il s'agit d'une crise de la capacité des pouvoirs publics à incarner l'intérêt général, pour d'autres de l'expression d'une demande de prévisibilité face à l'incertitude des évolutions urbaines, d'une appétence nouvelle à la prise de parole s'exprimant d'abord par l'opposition [Masboungi 2001], de la mise en place d'une démocratie participative ou simplement de la manifestation d'une volonté de repli sur les espaces de l'homogénéité sociale stigmatisée comme NIMBY.

De nombreux travaux de recherche montrent que les conflits et controverses ne constituent pas un dysfonctionnement de l'action publique mais bien une modalité généralisée de sa mise en œuvre. Ils représentent une des modalités de relation entre habitants et l'action publique territorialisée. Par ailleurs, controverses, conflits et même contentieux tendent à être intégrés à la mise en œuvre des projets et de la gestion publique. De nouvelles stratégies et techniques de participation, négociation ou médiation se focalisent sur l'acceptabilité des projets et des procédures d'aménagement.

Cependant les conflits ne semblent pas pouvoir être désamorcés, « résolus » par des procédures de participation, négociations placées en amont des projets. L'étape de la constitution de certains habitants en acteurs collectifs, de l'auto-proclamation de groupes comme représentants du territoire, d'implication d'habitants mobilisés et de "concernement» du public semble nécessaire pour qu'émergent des interlocuteurs de l'action publique. Ce processus peut être analysé comme la constitution d'une scène locale de débat au sein de laquelle il est possible de négocier et de construire les conditions d'un accord, d'un assentiment. Pour les habitants ou usagers, le conflit est un moyen de créer un rapport de force, d'obliger les acteurs publics à ouvrir une nouvelle phase de concertation. Même sans manifestation de conflit des associations utilisent le langage du conflit pour légitimer leur prise de position pour se poser comme acteur. Les conflits 
peuvent être analysés comme des formes de production négociée du cadre de vie urbain.

Il ne s'agit pas de réhabiliter le NIMBY comme porteur d'un bien commun localisé mais de considérer les mobilisations dans leur dimension d'action collective et de production territoriale. En effet, il faut souligner que le cadre d'analyse NIMBY mis en avant par les aménageurs fonctionne comme une stigmatisation des attachements de proximité [Trom, 1999; Thévenot, 2000]. Les habitants mobilisés sont soumis à une «contrainte d'universalisation » [Charlier, 1999: 262] qui se traduit par la nécessité de montée en généralité, de traduction ou de «transcodage » [Lascoumes, 1994] à partir de leurs revendications localisées et de leurs intérêts spécifiques. Prendre ses distances avec l'appellation NIMBY, n'implique pas de considérer les mobilisations habitantes comme l'émergence d'une démocratie participative de proximité ou comme une étape vers une mobilisation sur des thèmes globaux sous l'impact des processus de montée en généralité. Certains auteurs envisagent pourtant l'émergence de mouvements environnementalistes de base ${ }^{4}$ dont la généralisation révèlerait l'existence d'une véritable culture environnementale et de mouvements qui se situent à l'interface entre préoccupations environnementales et autres préoccupations sociales. Bruno Charlier évoque la constitution d'une écocitoyenneté [Charlier, $1999: 609]$.

Ce qui serait en jeu c'est la fin d'une vision transcendantale de l'intérêt général [Duarte, Novarina, 2000] et sa négociation au cas par cas comme conciliation de différentes formes d'intérêts publics et privés. Les projets des acteurs publics sont considérés au même niveau que d'autres intérêts, pratiques ou usages du territoire.

Le conflit ou même simplement les signes et le langage du conflit et de la controverse sont donc le moyen pour des groupes mobilisés de s'instituer comme interlocuteurs. Le conflit serait donc en cela une stratégie de conquête de l'espace politique public. Dramatisation de la relation entre les habitants, les concepteurs de projets et de procédures d'aménagement et les politiques, les conflits constituent des moments forts du débat public. Devant la faiblesse de l'espace politique local, de médias locaux offrant une scène pour la discussion de l'opportunité des projets, le conflit construit une scène locale de débat.

L'hypothèse centrale qui fonde l'intérêt d'une étude des conflits pourrait donc être formulée ainsi : par leur existence même comme scène de débat ou par la mise en œuvre d'instance ad-hoc de négociation de l'assentiment, les conflits contribuent à la production de ce qu'Alberto Melucci propose

${ }^{4}$ Cf. R. Strassoldo [1993] cité par Bruno Charlier [1999] propose la catégorie de MAB = Movimiento ambientali de base. 
d'appeler un espace public intermédiaire, nouvelle forme d'espace politique dont la fonction n'est pas d'institutionnaliser les conflits mais de construire les conditions de la prise en compte de leur discours et de leur transformation en décisions politiques. Dans le cas des conflits d'implantation ou d'environnement, cet espace public intermédiaire est territorialisé. Les mobilisations, conflits et leur prise en compte par l'action publique peuvent être analysés comme une expérience collective de coproduction de l'intérêt général [Latour, 1999] mais aussi comme une expérience collective de production territoriale.

\section{Bibliographie}

Blais J.P., Gillio C., Ion J. (animation) [2001], Cadre de vie, environnement et dynamiques associatives, Actes du séminaire, PUCA, $351 \mathrm{p}$.

Callon M., Lascoumes P., Barthe Y. [2001], Agir dans un monde incertain, essai sur la démocratie technique, Paris, Seuil, 345 p.

Charlier B. [1999], La défense de l'environnement: entre espace et territoire, géographie des conflits environnementaux déclenchés en France depuis 1974, Thèse de doctorat, Université de Pau et des pays de l'Adour, $753 \mathrm{p}$.

Coser Lewis A. [1982], Les fonctions du conflit social, PUF, Paris, 183 p. (première édition 1956).

Courty G. [1993], « Barrer, filtrer, encombrer : les routiers et l'art de retenir ses semblables », Culture et conflits, $\mathrm{n}^{\circ} 12$, hiver, p. 143-168.

Di Méo G. [1998], Géographie sociale et territoires, Paris, Nathan, 317 p.

Dziedzicki J.-M. [2001], Gestion des conflits d'aménagement de l'espace : quelle place pour les processus de médiation?, Thèse de doctorat, Université François Rabelais, Tours, 443 p.

EtiEnNe J., MENDRAs H. [1999], Les grands thèmes de la sociologie par les grands sociologues, Armand Colin, $256 \mathrm{p}$.

Fillieuele O. [1993], «L'émergence de la violence dans la manifestation de rue. Eléments pour une analyse étiologique », Culture et conflits, n9-10, printemps-été, p. 293-314.

Fillieule O., Pechu C. [1993], Lutter ensemble, les théories de l'action collective, L'harmattan, $221 \mathrm{p}$.

Fourniau J. M. [1996], «Les associations et le débat public», dans, Séminaire associations, PUCA, Ministère de l'Equipement, Paris.

GESSNER V. [1993], "Conflit», dans, Dictionnaire encyclopédique de théorie et de sociologie du droit, Paris, LGDJ, 758 p., p. 91-94.

ION J. [2000], «Introduction », Séminaire dynamiques associatives et cadre de vie, PUCA, compte rendu numéro 3.

LAfArgue J. [1998], La protestation collective, Paris, Nathan, 128 p. 
LASCOUMES P. [1996], L'éco-pouvoir, environnement et politique, Paris, éditions de la découverte, $317 \mathrm{p}$.

LATOUR B. [1999], «Introduction », dans, Jacques Lolive, Les contestations du TGV méditerranée, Paris, L'harmattan, $314 \mathrm{p}$.

LEGENDRE P. [1999], Sur la question dogmatique en occident, Paris, Fayard, $339 \mathrm{p}$.

LEVY J. [1994], L'espace légitime, sur la dimension géographique de la fonction politique, Paris, FNSP, $441 \mathrm{p}$.

LolIVE J. [1999], Les contestations du TGV méditerranée, Paris, L'harmattan, $314 \mathrm{p}$.

LuSSAUTL M. [2001], «Temps et récit des politiques urbaines », dans, Thierry Paquot (dir.) Le quotidien urbain, essais sur le temps des villes, Paris, p. 145-157, 191 p.

MARIE M. [1999], «Conflits d'eau, le jeu des antagonismes réglés entre SAR, Fermiers et collectivités locales en Provence », dans, M. Marié, D. Larcena, P. Dérioz (dir.), Cultures, usages et stratégies de l'eau en méditerranée occidentale, tensions, conflits, régulations, Paris, L'Harmattan, p. 239-246.

Masboungi A. [2001], "Du bon usage de la chronotopie », in, Paquot Thierry, Le quotidien urbain, essais sur les temps de la ville, La découverte, Institut des ville, 191 p., p. 167-189.

MeEus G. [1997], «La médiation, pour encadrer et résoudre les conflits du cadre de vie », Environnement et société, n 18, p. 45-48.

MelE P. [2001], «Habitants mobilisés et qualifications patrimoniales et environnementales », communication au séminaire du projet Qualification juridique de l'espace: structure de confiance de l'habitat, VST, CEOS, Lisbonne, novembre 2001.

Melucci A. [1999], Acción colectiva, vida cotidiana y democracia, Mexico, El Colegio de Mexico, 260 p.

Micoud A., Charvolin F. [2000], «La dynamique des associations de nature et d'environnement ", Séminaire, Dynamiques associatives et cadre de vie, PUCA, 2000, compte rendu n², p. 59-82.

MironesCo C. [1982], La logique du conflit, théories et mythes de la sociologie politique contemporaine, Paris, Editions Pierre-Marcel Favre, $185 \mathrm{p}$.

PiCON B. [1988], L'espace et le temps en Camargue, Actes Sud, 231 p.

Simmel G. [1995], Le conflit, Paris, Circé, 158 p. (première publication 1903 vérifier?)

STRASSOLDO R. [1993], Le radici dell'erba, sociologia dei movimenti ambientali de base, Naples, Liguori Editore, 386 p. 
THEVENot L. [2000], « Constituer l'environnement en chose publique, une comparaison franco-américaine », Séminaire Dynamiques associatives et cadre de vie, PUCA, compte rendu numéro 3, p. 5-21.

Touraine A. [1973], Production de la société, Paris, Fayard, 542 p.

TourAine A. [1984], Le retour de l'acteur, Paris, Fayard, 350 p.

Trom D. [1999], « De la réfutation de l'effet Nimby considérée comme une pratique militante », Revue Française de Science Politique, vol. 49, nº 1 , février, p. 31-50.

ZWETKOFF C. [1997], "Sentiment de justice et conflits d'implantations », Environnement et société, nº18 : 5-19. 